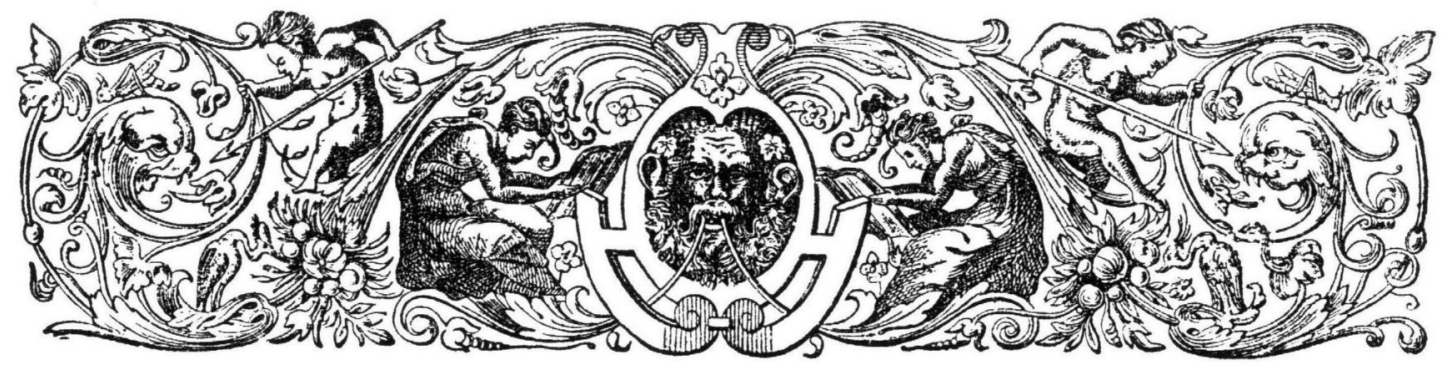

\title{
Gegevens omtrent Bouw en Inrichting van de Sint Bavokerk te Haarlem \\ DOOR
}

A. W. WEISSMAN.

II.

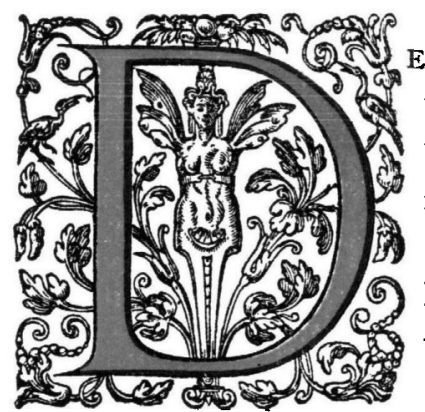

E belangstelling, die de gegevens omtrent de Sint Bavokerk te Haarlem, welke ik aan het handschrift van HENDRIK VAN ZUTPHEN ontleende, 1) vonden, noopt mij er andermaal een keuze uit te doen.

In $148 \mathrm{I}$ werd een belangrijke partij baksteen gekocht, zooals reeds in het kort werd vermeld. Men was toen bezig met het bouwen van den middenbeuk van het schip, en de steen werd gebruikt voor het achtermetselen van de "bloksteen", die door kerkmeesteren ter zelfder tijd werd aangeschaft. Hierop hebben de volgende aanteekeningen betrekking.

"Wi kercmeesteren hebben gecoft van een goet man gheheten HuBRECHT VAN VALCKENBURCH CL dusent steenen als wi van derselve steen hebben gehad van denselven HUBRECHT, elc dusent te leveren tot Hairlem voor XIII stuv. te betalen d'eene helft terstont end' andre helft tot Sint Baef's misse naestcomend."

1) Zie Oud Holland, I9r5 blz. 65 en vlg. 
nHebben noch gecoft ii dagen na St. Pytersdach ad vincula van HuYbrechT voirscr. $1 \times x v$ dusent steenen van derselfder aerde, maer met voirwaerde dat se een weynigh dicker wesen sullen dan die andere, want dat een nywe forme es, elc dusent voor XIII stuv. gheliic boven, met voirwaerden, datter gheen wanbacke steen bi es, want die sullen onse dienaers uutschieten moghen ende dat sonder berispen, te betaelen d' eene helft rede en d' andere helft tot St. Martijn ofte St. Cathrijn naestcomende.

"Wi hebben ontfanghen op onser Vrouwenavond Assumptio van HubrechT voirscr. XL dusent steenen en dair waren onder die in den gronde gheweest hadden. Noch hebben wi ontfanghen op onser kercmis avont van den voirscr. XIII dusent stenen min CC stenen ende in dese stenen was al te veel wanbacken steen in. Van vracht den scipper betaelt ii wilhelmus tuynen het dusent, dat is Ix stuv. en een oort.

„Wi hebben dese voirscr. stenen aen HUBRECHT betaelt, behoudens dat hi ons noch sal leveren xxxviII dusent stenen, elc dusent voir xIII stuv. en boven dit soe heeft hi ons gheloeft als een goet man dat hi alle de stenen die hi meer heeft dan de stede noch van hem hebben sal, ooc ons leveren sal, dairtoe sullen wi hem dan wat meerder affgheven".

De steenbakker HUBRECHT VAN VALCKENBURCH, waarvan hierboven sprake is, woonde te Leiden. Men ziet, dat er in de vijftiende eeuw op de hoedanigheid van te leveren steenen met veel zorg werd gelet en dat men toen geen ,wanbacken" of op andere wijze minder goed geworden steen aan een gebouw verwerkte.

De steenen werden in kalkmortel gemetseld. Want in $148 \mathrm{I}$ lezen wij: „Wi hebben ontfanghen daechs na hairlemmer kercmisse van een goet man uut Alcmaar xxxII hoet calc de hoet xv stuv., te betaelen op sint Matthysdach in den winter".

De baksteen diende alleen voor het achtermetselen van gehouwen steen. Die gehouwen steen is bedoeld in de volgende aanteekening. „Op St. Michielsdach hebben wi ontfanghen van JAN RUGHER ende GOOSEN RUGHER 500 voet blocsteyn opten eersten Saterdach in de vasten. Wi hebben eene overdracht met hem gemaect dat hi ons die voirscr. steyn leveren zal elc soo voir XIII rinse gulden buten die vracht."

Deze „bloksteen" schijnt de zoogenaamde „Gobertange” geweest te zijn, de harde witte kalksteen van de Maas, waarmede de muren der meeste kerken van Zuid. en Noord-Nederland in de vijftiende eeuw bekleed werden. Want een aanteekening van I48 I zegt: „Op St. Willeboirts dach hebben wi kercmeesters gerekent met WOUTER VAN RUGHEN eerst van de godelsteyn (Gobertange) die hi gelevert heeft int jaer 1479 naer uutwisen vant kerckeboec, ten anderen van de 
steyn welcke int jaer $148 \mathrm{I}$ de voet is gherekent voir sesthiendhalve stuver." Ik houd het er voor dat Wouter vaN RUGHEN, die ook wel RUGHER genoemd wordt, een broeder was van GozewIJN RUGHER en een zoon van JAN Rugher, een steenhouwer, die te $Z$ wolle woonde, en ook veel aan de Domkerk te Utrecht heeft geleverd. Te Utrecht ontving men, behalve „bloksteen”, ook veel geheel bewerkte steen, die alleen maar gesteld behoefde te worden. Ook te Haarlem was dit het geval, zooals uit de volgende aanteekening blijkt.

"Wi kercmeysteren van Haerlem hebben betaelt Wouter vaN RUGHeN in den jaire van $8 I$ in der maent Mey xviii $R h$. gul, tot afcorting van van de boechsteyn, die noch after de cellebroers an die noirtside en ant westent van der kerc staet en noch ongemetseld is, voir welcken ghelt WOUTER voirscr. ons heeft gelevert twe halve cappeteelen int jaer 80, welcke opter cruyspilaernen sullen staen boven St. Bavo ende St. Willeboirt".

Wij zien dus, dat in I48I "boogsteen", - afgewerkte steenen voor bogen -, door VAN RUGHEN was geleverd. Welke bogen hier bedoeld zijn, meldt de aanteekening echter niet. Zij waren nog "ongemetseld" en de steenen waren aan de noordwestzijde van de kerk opgestapeld. De steenhouwer leverde toen ook de "halve kapiteelen" die zoude komen te staan "boven St. Bavo en St. Willebrord". De beelden dezer heiligen waren tegen de westelijke pijlers van het koor geplaatst. Men ziet de "halve kapiteelen" daar nog; zij dragen de bogen, die over den kruisbeuk geslagen zijn. De muren van het koor zullen dus in $148 \mathrm{I}$ nog niet hooger opgetrokken geweest zijn.

De "halve kapiteelen" komen nog eens voor in een aanteekening.

Alsoe Steven Elens en Govert die Bosscher die twe halve cappeteelen nyet ghelevert en hebben, mits dat STEvens Elens doet es en Govert diE BosSCHER uten lande van Brabant gebannen es en die boechsteyn die noch ander noertside en 't westend van de kerc staet den voirscr. GOVERT DIE BOSSCHER, Stevens Elens en Wouter van Rughen tsamen toebehoirt, soe sullen wi kercmeesteren de voirsc. XVIII Rinse gul. afcorten en een betalinghe wesen aen de eerste van de ghelde die wy van de voirscr. boechsteyn geven of betalen sullen. Dese cappeteelen van de halve pylairnen aen het cruyswerc den voirmelden besteet synde om gheliic mette boechen te betaelen die after in die kerc staen'.

Het was dus met deze steen een vrij ingewikkelde zaak. Zij behoorde aan Govert DE Bosscher, Steven Elens en Wouter van Rughen gezamenlijk. Elens was dood, Govert was uit het land van Brabant gebannen en men wist blijkbaar niet, waar hij zich ophield. Daarom werd Wouter VAN Rughen als de eigenaar beschouwd en men deed hem een betaling. Hoe het verder gegaan is, vinden wij in de aanteekeningen niet. 
In 1485 komt een andere steenhouwer in de aanteekeningen voor, namelij Jan Bouwens, die kreeg "Ix Ryns gul. van de derde boech, en noch Ix Ryns. gul. van de vierde boech ende noch Ix Ryns gul. van de vijfde boech en vant harnass in dat grote glas en anderen xc Ryns gul. ende twe stuvers."

Dit zijn waarschijnlijk de bogen aan de noord- of zuidzijde van den middenbeuk en het venster aan de westzijde der kerk, wat toen blijkbaar onderhanden was, tenzij met het "grote glas" bedoeld mocht zijn dat in den zuiderkruisbeuk, omdat wij in 1486 vinden: "DIRC JACOPS die beeltsnider gegeven van de $\mathbf{x v}$ loveren te maecken die op het suder cruyswerc staen Io Ryns. gul. ende 8 stuv." In 1488 stond het beeld van St. Bavo reeds in den gevel van den zuider kruisbeuk, want toen kreeg "CLAES vAN WATERLANT voir dat verwen en verghulden van Sint Bavo in den suder ghevel $12 \mathrm{Rh}$. gul." Men was toen ook bezig met het dekken van de kap, want er werden geleent ${ }_{n}$ van den Guardiaen vant Convent den Minrebroers van de Observancie binnen Hairlem XXVIII hondert leyen tot het decken."

In 1499 begonnen de werkzaamheden tot het overwelven van het middendeel der kerk. Dit overwelven, hetwelk in I 500 gereed kwam - zooals een opschrift in de kerk nog doet zien - geschiedde in steen. Hierop hebben de volgende aanteekeningen van 1499 betrekking.

„Den XXIII Septembris wesende een Maendach is in presencie vande IV kercmeesteren en POUWEL JANSz. aenghenomen doer JAN DiRICS en HeYNRIC DIE HAESE dat harniss twisken die twe cruyswercken van dat wolleft ende sullen dat harniss macken by de voet voir ii stuyv. uutgenomen die slotsteynen, die sullen se wercken bi den dach en sullen beginnen daechs na Alderheyligendach en sullen daervan niet uutscheyden voir het werc ghereet ende wel ghemaect is".

Dit waren de ribben van het stergewelf, die van gehouwen steen werden gemaakt. De baksteen, die voor de schilden en de aan aanraseering van het gewelf zou dienen werd "ghecoft van Dibbout Harmansz tot Leyden". Hij leverde "L dusent leytsche steenen het dusent voir XV stuv."

Van Pouwel Jansz. - misschien dezelfde, die reeds vroeger werd genoemd - kocht men 50 hoed kalk, ,het hoet XVI stuvers'. En dan zegt een aanteekening: "Simon Maerten Cornelisz sal ons soe veel sant brenghen als noedich es omme daermede $\mathrm{L}$ hoet calc te beslaen en daerenboven dat bedde van sant daer men de calc op beslaen sal en als se beslaghen is soeveel sant om te decken. Hiervoir sal hy hebben xl stuvers en voir het beslaen van de calc I stuv. voir de hoet".

Het was in I 499 , toen men aan het gewelf boven het kruis der kerk begon, reeds de bedoeling, daarop een toren te plaatsen. Want een aanteekening zegt: „JACOP DIE WAEL heeft der kerc geloeft indien dat men voirt tymmerde aen 
de bellefroed ende men maecte een voirslagh, soe geeft hy dairtoe die groetste cloc ende swaerste van ghelut."

In 1500 was het gewelf gereed. „PYTER WILLEMSZ. scilder sal ons verwen dat wolft midden in der kerc mit bloemen ende ooc gout legghen dair hi sulcs van doen heeft en hi sal ons het goet macken dairvoir sal hi hebben voir synder arbeyt en voir synder hulp DIRCK ende JOEST. Hi sal ons ooc alle die slotsteynen verwen en gout legghen dair sulcs wesen moet. Dairvoer sal hi hebben XVII Ryns gul. en dat gout sullen wi betaelen."

Het schijnt echter, dat WILLEMSZ. het versieren der sluitsteenen niet heeft verricht, daar een aanteekening zegt: „ClAES YAN WATERLAND heeft ons geverwet alle de bloemen die onder aen de slotsteynen syn aent wolft mids in die kerc ende daer syn xii hele ende iv halve en een groote middenin. Hiervoir sal hi hebben van verghulden xviii Ryns. gul."

Om de kruispijlers, die den toren zouden dragen, en die niet met dit doel gebouwd waren, te versterken en met elkander te verbinden, maakte JACOP DIE SMIT vier ijzeren ankers.

En, ofschoon er nog geen toren was, „besteedden kercmeysteren opten IV Septembris I 500 aen meester JAN GHOBELSZ wonende tot Delff twe clocken die sullen weghen 1500 off 1600 pont ende moeten dienen op onsen toirn. Hi sal se ons tot Hairlem leveren op sine costen, wi sullen hem die stoff leveren op onse waech en dese clocken sullen ghemaect syn voir alderheyhighen naestcomende. $\mathrm{Hi}$ sal hebben van de $\mathrm{C}$ pont xxxiii stuv. indien dat si ons dienen sullen gheliic dat bestec uutwist dat light in der kercken lade."

In 1500 werd het beschilderen der luiken van het hoofdaltaar aanbesteed aan Jan Mostaert. Buiten op de luiken moest hij maken "Onse lieve Vrouw" en "St. Bavo", ieder "mit een tabernaeckel daerboven". Van binnen moesten komen: „XII parcken mitter storye van St. Bavo wel ghewerct ende ghemaect soe dat wi reden sullen hebben hem te bedancken." MOsTAER'T zou "als 't werc volmaect" was 20 Rynsche guldens krijgen.

In 1501 werden bouwstoffen voor den toren aangeschaft. De kerkmeester JACOB ENGBRECHTSZ ging toen naar $Z$ wolle, en kocht daar den 6den September , in presencie van JAN VAN SwOLL en van de waert int Moriaenshoeft van HENDRIC VAN NyENHUSEN voir die kerc dusent voeten bentemmersteyn en sal se ontfanghen tot Swoll ende als hi self dair nyet en is dan sal hi se bestellen aen sinen waert tot Swoll, genaempt GHeryt ScEePker in den Gulden Hollantsen Tuyn, het hondert voir vii Rhyns. gul."

Deze steen werd door den schipper DIRC MEYNerTs naar Haarlem vervoerd; men betaalde hem 22 stuivers voor de honderd voet. Als verdere onkosten worden 
nog vermeld: "Aen die ghesellen die in de craen de steyn opwynden ii stuv. voir die C voet."

"Voir het slepen van de steyn opt nyewe kerchoff xix stuv."

"Aen die gravemaeckers voort optassen van den steyn vil st."

"Voir het slepen van de steyn op 't nyeuwe kerckhoff xix stuv".

"Aen die gravemaeckers voort optassen VIII st".

Den $9^{\text {den }}$ September 150 I werden van CoRNelis LOEFFsz te Leiden 200.000 baksteenen gekocht, die per 1000 een en twintig stuivers en een oort kostten.

In 1502 werd met het werk begonnen. Eerst moest men een voet maken, die de vier kruispijlers met elkander verbond, en die den toren kon dragen. Het ontwerp voor dien voet werd gemaakt door CORNELIS DE WAEL, die toen met het bouwen van de Domkerk te Utrecht bezig was. Men vroeg er ook het oordeel over van "Meester ANTONys Kelremans, bouwmeester van Mechelen". Het "patroen daer men naer wercken sai mydden op de kerc" werd door DIRC TyвouTsz van hout gesneden. Maar het schijnt, dat dit model niet voldoende werd geacht, immers wij vinden in 1502 aangeteekend : „PAULUS JANSZ onse metselair gesonden totten meester te Wtrecht om met hem te spreken van der kerc ende berderen te halen daer hi naer wercken sal, verteert heen en weder thuus en metten Meester te schenken XXIV stuv. ende voir syn daggelt $V$ daghen XXVIII stuv".

De uitvoering van het steenhouwwerk geschiedde door JAN DIRICs en HENRIC DIE HAESE. $Z$ ij namen aan „dat harniss ende arcaturen te maecken van den voet boven de kerc na uutwising van dat bort dat dairvoir ghemaekt es". Hun loon zou zijn ,ii stuv. min een oortgen van den voet". En ,indien dat sy se maecken datse nyet en sluten als men ze plaetsen sal en zoe er enich ghebrec aen is dan sullen si se te hoeren cost vermaecken".

Toen dus de voet van den toren begonnen was ging men klokken bestellen. „In manieren hierna ghescreven hebben die burghemeesteren mette kercmeesteren besteet meester GHERYT VAN WOU die clocghieter tot Campen een uyrcloc weghende tyen dusent ponden oft daeromtrent, van onse stoff, scoen van ghelut al sonder fault die die stemme hebben sal van XII dusent pont in ghelicke resonancie soe de uercloc heeft tot Ghent, als hi dat aldernaest raken can en heeft gheloeft daeromme tot Ghent te reysen omme die maet daeraft te nemen opdat hi te sekerder ons daerin voldoen mach. Ende wairt dat hier enich ghebreck in geviel, dat zal meester GHERYT voirscr. ons beteren ten sinen costen. Ende voir dese voirscr. cloc sal hi hebben voir sinen loen van elc $C$ pont xxxv stuv, ende hyer boven zal men hem leveren leem, het gereetscap en de arbeitsluden die hiertoe behoeftich zullen zyn, wel verstaende dat hi ende syne 
knechten zullen zyn ten lasten ende costen van der kerc. De burghemeesteren ende kercmeesteren hebben noch meester GHERYT besteet vi off viii clocken tot een voirslach, alsoe groet als men hem dan zegghen sal als die groete clock voircrs. gegoten es. Dese vi off viii clocken van dit voirslach sal meester GHERYT gieten tot Campen van syn selfs stoff op hetzelfde accoert sonder fault op malcander alsoe dat behoirt en sal voir yder hondert pont hebben ix guldens en sal dese clocken leveren tot Hairlem inde waech, de groete cloc sal hi ghieten tot Hairlem twisken Paschen ende Pynxteren in het jaer XVc ende iii".

De uurklok, in deze aanteekening bedoeld, is inderdaad door GERRIT VAN Wou gemaakt. $\mathrm{Z}_{\mathrm{ij}}$ doet nog altijd in den toren dienst. Maar het klokkenspel leverde de beroemde gieter niet.

"Int jaer XVc ende III 20 hebben wi kercmeesteren gherekent met meester Gheryt vaN WoU, clocgieter tot Campen van die uyrcloc die hy der stede gegoten heeft ende gehele somma van der voirscr. cloc was in alles I400 Rhyn. gl. current waervan hi ontfanghen heeft dry hondert Rhyn. gl, en de andere $1100 \mathrm{gl}$. in VIII terminnen sal ontfangen, zoe dat die voirscr. cloc op St. Bavos misse I 507 betaelt zal zijn".

VAN WoU leverde dus ook de klokspijs, ofschoon aanvankelijk bedongen was, dat de kerkmeesters die zouden leveren. De groote gieter was geen best man van zaken, daar hij er in toestemde, dat de betaling zoo lang werd uitgesteld. Het is te begrijpen, dat onder deze omstandigheden van het leveren van het klokkenspel niets is gekomen.

Trouwens, met den toren ging het veel langzamer, dan verwacht was. Wel vinden wij aangeteekend in 1503: ,Meester CoRNELIS van Utrecht, onse meester van den wercke, quam tot Hairlem opten X May, synde een Donderdach om ons werc te besien, dat hem seer wel behaechde en was alhier IV daeghen ende schonken hem en ooc die met hem quamen teren als van den gerechte ende kercmeesteren ende andere goede mannen, die hem selscap bielden en voeren met hem op zee".

CORNELIS DE WAEL werd goed onthaald; de heeren van den gerechte en de kerkmeesters gaven als kosten op: „Verteert in dien tyt aen maeltiden ii $\mathrm{Rh}$. gl., aen wyn iii $\mathrm{Rh}$. gl. en viii stuv. ende daerenboven verteert op zee ende aen den Heylighen Berch met spyse die wi namen ende met wagenhuyr XXIII st." Hieruit valt op te maken, dat de reis van Utrecht naar Haarlem over Amersfoort ging, waar men zich op den Heiligen Berg schijnt te hebben opgehouden. Te Amersfoort gingen de reizigers vermoedelijk in een schip de Eem op, toen de Zuiderzee en het IJ over, om door het Spaarne Haarlem te bereiken. Maar de terugreis naar Utrecht ging over Leiden; daarvoor werd aan CoRNelis 
DE WAEL 2 Philipsguldens van 25 stuivers betaald. Als honorarium ontving hij 5 Philipsguldens.

In I 505 was CORNELIS DE WAEL gestorven. Als zijn opvolger werd toen, zooals reeds vroeger werd medegedeeld, AnTHONIE Keldermans uit Mechelen benoemd. De lotgevallen van den toren hebben wij ook reeds vermeld. In I 5 I 6 moest die worden afgebroken, omdat hij te zwaar voor de pijlers was. Wat is er met die afbraak gedaan? Voor de kerk, die toen voltooid was, kon men haar niet gebruiken. Ik veronderstel, dat men er het bovendeel van den toren der Bakenesserkerk van gemaakt heeft, en dat dit bovendeel dus nog de herinnering aan den toren van KELDERMANS bewaart.

Omtrent de orgels der St. Bavokerk geeft de volgende oorkonde bijzonderheden.

„Wi Ghildebruers van onser Lieven Vrouwen Ghilde der Kercke van Hairlem, die hair namen met hair selffs handen hier onder gescreven staen, verclaren voir die gherechte wairheyt ende segghen, dat ons wel sculdich is, dat doe ter tyt ter begheerte der Stede van Hairlem geconcludeert ende gheconsenteert was bi die Deken, Scrivers, Vinders ende Ghildebruers voirsz. om een nuwe Orghel te maecken met nuwe verscheyde gheluide in de zuutside van de voirscr. Kerc alst ghemaect is. Dat men d'oude Orghel, staende aen die noirtside, onser Lieve Vrouwenghilde mede toebehorende, vercopen soude tot hulpe van die nuwe mede te betalen. Ende dat naderhand die Kercmeesters bi twisken spreken van die Burghemeysters eene contract mit die Deken ende Scrivers ghemaect hebben, dat die oude Orghel voirscr. die als voren dat ghilt toebehoirde, soude in de kerc bliven en waert, zoe 't behoefde ghereformeert te wesen, dat ghilt sout doen reformeren als propritarisse om daechlix ende opten hoechtiden tot voortcominghe vant nyewe werc te spelen, waertoe die kercmeesters dat ghilt te hulp zouden comen mit twyntich pont vlaems, te betalen als dit tegenwoordighe instrument ofte copia gecollacioneert bij Meester ClAES BOOT secretaris der Stede van Hairlem verclaert. Ende overmits dattet nyet dienen en zoude dat men daechlix op sulcken sumptuosen ende subtylen wercke spelen zoude en zoe schrael soude maecken ' $t$ welc alle die ghemene ghildebruers nyet goedt dachte en noch mishaecht dat sy daechlixs daer zoe veel op spelen ende zoe schrael maecken. Ende segghen mede dat mit reformeren MR. JAN dat werc in zyn gheluide vercort heeft, als een yghelic kundich is dat te voren was."

Het orgel aan de noordzijde, dat hier bedoeld is, bevond zich in den zijbeuk van het koor, boven de kapel van Schagen. De acte, die den gden September 1523 werd opgemaakt, zegt, dat men het toen reeds had willen verkoopen, maar dat het op verzoek van Burgemeesteren door MR. JAN was hersteld, opdat

Oud Holland, 1916. 
het zou kunnen worden bespeeld zoolang het nieuwe orgel, dat in den zuiderbeuk van het schip zou komen, niet was gemaakt. Het schijnt, dat de orgelmaker niet gelukkig met zijn werk was geweest, daar het instrument na de herstelling minder geluid gaf. En het Lieve Vrouwegild, dat de Kapel van Schagen en het orgel bezat, vond het voor het orgel niet goed, dat het iederen dag werd bespeeld.

Het nieuwe orgel aan de zuidzijde van het schip kwam in $1527 \mathrm{klaar}$, hetwelk uit de volgende acte blijkt.

„Wi Meester Cornelis Claes ende Martyn Wolfaerts Vinders van Onser Lieven Vrouwenghilde bekennen ende beliden ontfanghen te hebben van die kercmeesters van der Parochykercke binnen dezer Stede die Somma van Veertich Ryns. Guldens tot $\mathrm{xl}$ gr. vlaems 't stuc in betaling vant leste termyn $t$ welc de voornoemde kercmeesteren sculdich waren te betalen van den cleyn orghel na inhout 't ynstrument daeraff wesende van welc termyn van veertich gulden en pryse voerscr. met die ander twe termynen wi ons houden vernoucht ende wel betaelt, sceldende de voirn. kercmeesteren daervan quyte en alle anderen die 't behoeren zal. 't Oirconde onze gewoenlycken hantscriften hyer onder gescreven opten twyntichsten dach novembris int jaer ons Heren duyzent vyffhondert zeven en twintich".

In I 524 werden de klokken besteld van het "voorslag", dat in den toren geplaatst zou worden.

"Daechs nae Sint Jansdach syn gheboorte Anno XVc ende XXIV syn de kerckmeesteren van die kerc van Haerlem overghecomen mit meester CoRNELIS WAGHevens van Mechelen als dat hy die kerc leveren sal elff clocken op haer toen ende accoerden tot een voirslach alsoedra als hi mach ende die te Mechelen te leveren twysken dit en Pynxter naestcomend waervoer dat hondert pont die kerc hem betaelen sal XIV Rynse gulden curant ende vii stuyv. en dat te weten een vierendeel ghereet te betalen op Alderheyligen ende dat ander deel soe hi levert ende dat derde deel een jaer daernae ende dat vierde deel wederom een jaer daerna. En wacr 't saecke dat in die betalinghe enich ghebreek viel zoe zullen wi dat stellen te lossen de penning zestien ter tyt toe 'jaers te betaelen den voirscr. Mr. CoRnelis tot die renten die verlopen syn met het hoeftgelt betaelt zal wezen. En dit op die noet $\mathrm{Fa}$ als tot Antwerpen is op die toren van onze Lieve Vrouwe tot vyff dusent pont toe of dair omtrent weghende ende soe wat clocspys is dat wi hem leveren sal wesen tot affslach".

Reeds werden eenige mededeelingen gedaan over deze leverantie. Wij laten nu de aanteekeningen, die op dit klokkenspel betrekking hebben in haar geheel volgen.

In 1525 vinden wij: ,Meester ClaES GlasenMaECKER en de Sancmeester 
VAN Mechelen en woent bynnen Hairlem hebben tsamen te Mechelen geweest omme te ontfanghen die $\mathrm{xi}$ clocken tot dat voirslach en syn nyet geweest als si behoerden van accoert en van gewycht. Vertert opten reyse ende voir haer suymenis en voer haer moeyte ghegeven XII R. guld".

De klokken voldeden dus niet. Later in 1525 ging JAN VAN MECHELEN nog eens kijken. "Meester JAN die sancmeester vaN MECHELEN gegeven omdat hi tot Mechelen wesen wylde en daer wat van doen hadde van syn selfs dinc en om naer ons werc te vernemen vant voirslach, nyet uut onsen naem, maer omdat hyt gedaen heeft die summe van Xxv stuyv."

In 1526 gingen eenige kerkmeesters weder naar Mechelen. "Meester CLAES Glasemaeker ghegeven van syn reyse doe hi tot Mechelen was mit Pieter JANSZ ende JAN NANNINGS als kercmeesteren om het voirslach te ontfanghen, voer $\sin$ verzumenis van die ghetijden en $\sin$ reysen en voir sin moeyte iii $R$. G. Meester JAN die sancmeester VAN MECHELEN is mede gheweest op derselve reyse, voir verzumenis van de ghetiden en sin sancmis en moeyte ghegeven iii R. G. Noch Meester JAN van een laedge daer die toen of noet in lach en van een pen IV stuver en een oirt'.

Wat men te Mechelen vond vermeldt de volgende aanteekening. "JAN Nannings Brouwer, Pieter Janszoens als kercmeesters, Meester Claes GlaseMAECKER, Pryster, syn gheweest den IV dach Junius anno XXVI te Mechelen ende ooc met Meester JaN de Sancmeester binnen deser stede en is van Mechelen, omme de XI clocken te horen off se goet waren van accoert als wi se CORNELIS WAGHEvENS van Mechelen aenbesteet hebben. Zoe als sy daer ghecomen syn soe is er nyet meer gereet geweest dan zeven. Dese voirscr. VII clocken weghen tot Mechelen 605 I ponden wairvan de kerc geven zal om te betalen tot 5200 pond voir die 100 pond I4 R. G. van 40 groet en 8 stuyvers en voir het hondert dat het meer beloept dan 5200 dat zal men betalen met elff R. G. het hondert van 40 groet, dus soe sal hi noch leveren iv clocken op die torn, die te cort ghelevert zyn het 100 voir elff R. G."

"Vande seven clocken van Mechelen tot Antwerpen van vracht ende van thollen 8 R. G. dit is bynnen Mechelen betaelt en noch van thollen gegeven t'Antwerpen 6 R. G. Voir het uut het clocghytershuys sceep brenghen betaelt I 8 stuyv. en te Mechelen en te Antwerpen met haer vijfven verteert I4 R. G. Verreyst en verteert met haer vyfven twysken Haerlem en Antwerpen heen ende terug 19 R. G., dus voer die VII clocken en alles 828 R. G. 9 stuv. en 15 duyten."

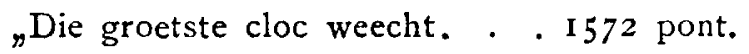

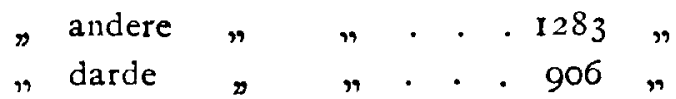


Die vierde cloc weecht . 874 pont

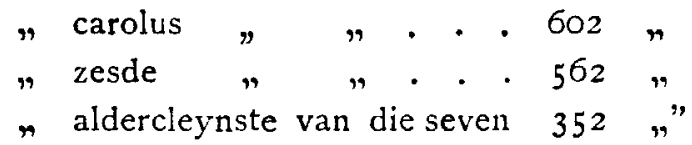

Zoo had men dus nu zeven klokken. CORNELIS WaGHEvens kwam eindelijk ook met de vier andere klaar. "DiRC JANSz KETELbOETER is geweest te Mechelen om zyns zelfs bedryf ende wi hebben hem macht ghegeven dat hi die vyer clocken mede brenghen soude alzoe verre als se ackordeerden. Zoe is hy gegaen tot Broessel tot een gheheten Meester Damiaen en is Sancmeester tot Broessel die sou die vier clocken horen of se goet van ackoert waren. Hi heeft ze goet geackordeert ghevonden, maer hy heeft nyet geacht dat wi hem gescreven hadden, dat was die toen of noet van deze clocken. Hi screef dat hi se wech gebracht had, daer hi se behyelt alst daertoe quam dat hi de clocken horen soude. Soe heeft meester DAMIAEN geseyt: had ic nu gheen beter besceit dan ghi mi brenght soe moecht dit laten bliven daert was of dierghelyc. Hyer mach men mercken dattet nyet en is alst behoert."

CORNELIS WAGHEVENS bood nu aan, de klokken voor minder geld te geven. Maar de kerkmeesters weigerden ze aan te nemen. „Betaelt AERNT VAN TUYLL van een brieff te brenghen tot die maker van die clocken dat ons die clocken nyet en dienden en een andere weder gebracht 14 stuv."

Men trachtte nu elders klokken te koopen. „Betaelt en ghegeven van de 2 clocken die wi van Amsterdam deden comen om te proberen ofte die ackordeerden, van deze clocken opslepen in de kerk en aen een man die de clocken mede op de wal en in der kerc bracht 5 st."

Maar deze klokken voldeden niet. „Betaelt van de clocken weder sceep te brengen alsoe se nyet en dienden en van vracht tot Amsterdam te brengen 6 st."

Eindelijk slaagde men beter. „Opghedaen uyt een scip komende van Berghen 3 clocken toebehoirende GHERYt KeTELboeter te Amsterdam om die te proberen I st. ghegeven den scipper die de clocken inhad dat hij hier toefde 2 st. Een dragher die de clocken in de kerc hielp brengen I st. Als wi die voirscr. clocken ghecoft hadden gebracht uut der kerc in die waech om te weghen van 2 mael slepen 2 st. Aan die hielpen de clocken in den toirn te brenghen 15 st."

Het schijnt, dat deze klokken te Amsterdam waren geladen in een schip, dat van Bergen in Noorwegen was gekomen, en dat ze voorloopig te Amsterdam gekeurd waren. Immers wij vinden: „Betaelt meester JAN die sancmeester van twe reysen te reysen tot Amsterdam om die clocken te soecken tot het voirslach 10 st. en een blanc."

Bijzonderheden omtrent de klokken geeft de volgende aanteekening: "Opten 
29 Mey 1527 is JAN NANNINGS kerckmeester ghegaen nae Amsterdam om te betalen de clocken die gecoft waren van GERYT KETELBOETER, verteert en van reysen 2 st. en een blanck."

${ }_{n}$ Betaelt voir drie clocken die woghen 674 pond de 100 pond gekoft voir I 5 gl. en wi hebben daeran ghelevert 270 pond spys dat 100 pond I 2 gl. dies hebben wi hem toeghegeven voir die reste $72 \mathrm{gl}$. $12 \mathrm{st}$. dairinne is gherekent voir die clock die men hier proefde en hier gestuurt worde $4 \mathrm{gl}$, makende tsamen die voirscr. som. Actum opten 25 Mey I 527 ".

De ontbrekende klok werd in 1535 besteld. "GHOEBEL ZAEL uut den Hage ghieter heeft een clock voor de kerc aenghenomen te ghieten van $\mathrm{CC}$ pont goet van gheluut dat Mr. JACOP onse sancmeester en onse beyerman segghen sullen dat hy voldaen heeft. Hij sal hebben een stuyver vant wisselen vant pont en dat hy daerby doet 3 stuyver en in dien sy niet goet is sal hy een ander leveren. Hierop hebben wy hem op die hant ghegeven $8 \mathrm{R}$. G. op Sint Marie Maddalenen avont. In kennisse der wairheyt zoe heb ic GHOEBEL ZAEL myn naem met myns zelfs hant gheset en zal dit leveren twysken dit en Sint Bartelmeus".

In 1534 werden er belangrijke werkzaamheden verricht aan het groote orgel der kerk; Meester CLAES was daarmede belast. Hem werd den $9^{\text {den Januari }}$ 6 gulden op rekening gegeven; den $25^{\text {sten }}$ Januari kreeg hij 7 gulden, den $27^{\text {sten }}$ Februari 40 gulden. En den I4den Mei werd betaald, aen des orghelmakers wyff op begeert van Mr. CLAES den orghelmaker op rekeninghe" 6 gulden.

Dit orgel bevond zich aan de noordzijde van het koor. Men maakte, „een nuywe camer daer de blaesbalcken in sullen legghen". Ook an de orgelkas werd gewerkt, en er kwam ,snywerck beneden aen den orghel". DAMIAEN de beeldsnyder kreeg "op Pynxter van sin arbeytsloen dat hy aen den orghel ghesneden heeft boven aen de tabernaeckel en midden voir die grote lade en beneden aen de foey" een som van 4 gulden. En "op Sint Jansavont wert JaN ENGBERTZ de blaser gegeven 4 Philips gulden voer dat hy van Sint Jorisdach af den orghelmaecker by het maecken vant orghel heeft geblasen".

De „verscheyde verven die PYETER JANSZ stoffeerder" bij het schilderen van de orgelkas gebruikte, werden bij "Comen TyBOUT DIE HAESE" voor 2 gulden en 8 stuivers aangeschaft. En van "URBAEN DIE Goutslager aan de Hal” kocht men 800 bladen goud voor 27 stuivers per 100, benevens 25 bladen zilver, die tezamen 2 stuivers kostten. Maar er werd veel minder verguld, dan oorspronkelijk bedoeld was, want de kerkmeesters zeggen: "ons is weder ten profyt ghecomen 550 bladen gouts, die aent orghel nyet ghebruyckt syn". PyeTEK Jansz kreeg als loon de aanzienlijke som van $\mathbf{I} 7$ gulden. 
In $153^{8}$ begon men met de werkzaamheden aan den westelijken gevel, die noodig waren om een gebrandschilderd glas, dat GEORGE VAN EGMOND, Bisschop van Utrecht aan de kerk wilde schenken, te kunnen plaatsen.

„Anno I 538 daechs nae conceptionis Marie hebben wij kerckmeesters voer intituleert aenghenomen ARENT HARMANSZ steenhouwer achtervolghens de nae te melden voirwaerden ornt harniss vant groete glass in de westerghevel te maeken en hebben met hem twe meesterknechts mede aanghenomen".

"Sy sullen wercken daechs van nu en voirtaen xiv daghen voor kercmis 1538 van des morghens dat men sien can tot s avonts in de late avont en sullen twysken dit en half vasten daechs hebben $V$ stuyver en nae half vasten zullen sy des ochtends ten $\mathrm{V}$ uyre beghinnen te wercken tot des avonts VII uren en omt eten telckens een half uyr en sal als dan des daechs wesen VII stuyver en sullen aen dat werck nyet uutscheyden voir dat het volbraght zal zijn buiten consent van de kerckmeesteren."

Dit stuk is belangrijk voor de kennis van het loon en den arbeidsduur in de eerste helft der zestiende eeuw. Als meesterknechts werden aangenomen JoEsT VAN BAMBEECK en JOEST JANSZ van Amsterdam ,dewelcke wekelyx haer loen ontfanghen zullen."

In 1539 vinden wij het volgende, dat een kijkje geeft op een meer huishoudelijk werk van die dagen. „Die kerckmeesters sullen gelieven te weten dat ic Marytje Pyter Jansz wyff die tralye die croenen en blaekers 2 mael in een jair sal scuren en het font, die pullicaen en hair blakers 4 mael tjaers ent goet int sacristy mette reliquien en het goet voir Sinte Baef alle viertien daech; die candelaers op thoge outaer alle lieve Vrouwe avonts, die armen van Sinte Baef en die 2 blaekers voir onse lieve Vrou in die son 4 mael tjaers en die tralyen 4 mael tjaers te raghen. Item noch sullen die kerckmeesters weten alles soe wat hyer an sal gebreken dat sullen si my an die pennynghe corten en ist saecke dat dair yet gebroken wert doir myn versumeniss ofte onachtsaemheyt dat sal ic laten maken op mynen cost".

In 1539 waren de werkzaamheden aan den westelijken gevel zoover gevorderd, dat men het ijzerwerk voor het groote venster noodig had. Daarvoor werd dat uit het vroegere venster versmeed, gelijk de volgende aanteekening leert.

„Daechs na onser Lief Heeren Hemelvaertsdach heeft WOUTER KERSTENSZ kercmeester gelevert in die waech doer EVERT die smit 2612 ponden yser dat ghecomen is uut dat oude glass van de westgevel der kerc. Dat yser sal EverT versmeden voir het nyewe glass in den westghevel met al hetgene dat daer verder aen geen sal, soe die wyndysers als die balcken dat hij weder sal leveren en het yser boven die voirscr. ponden bedraghende zal de kerc kosten voir het pont 
elff pennynghen. Hier teghen gelevert op Sint Marie Magdalenasavont 2975 pont, dus comt in afrekeningh $3 \sigma_{3}$ pont. Noch soe heeft hi aen de balcken in de westgevel ghelevert 224 pont yser".

In 1540 kwam een afgevaardigde van den Bisschop van Utrecht te Haarlem, om den kerkmeesters het besluit der schenking van het venster mede te deelen. Men gaf hem een vereering. "Gegheven den I 3 den Mey 1540 aen Mr. JAN DE BouvigniE kanonyk in den Doem 't' Uytrecht als Suffragaen van onse ghenadigen ende alderwaerdichsten Heere van Utrecht Biscop JoRvs vaN EGMONDT X Ryns gul".

Het duurde tot I54I eer met het glas een begin werd gemaakt. Den 30 sten Juni van dat jaar "quam Mr. GERyT BoELs glasemaecker tot Loven om die maet van glass in die westghevel te nemen, synde het glass van onse genadechsten en alderwaerdichsten Heer Biscop van Utrecht. Hem ghegeven V. Rijns Gulden ende betaelt syn costgelt tot IJSBRANT JACOPS Wed. xx stuyv".

In 1543 , toen het glas gereed was, liet men ook de glazen in den kruisbeuk herstellen. „Betaelt aen DiRCK WiLlemsZ glasenmaecker van het glas van den graef van Oistervant int noerder cruyswerck te vernieuwen en vant crucifix in twee panden gemaeckt van Hertogh Philips glas in het suyder cruyswerck".

In den toren der kerk hangen looden klokken, die slechts als versiering dienen, daar het klokkenspel niet zichtbaar is van buiten. Die klokken dagteekenen van 1587. "Aen ADRIAEN JANSz voor 8 looden clocken welcke in den thoorn syn opghehangen wegende tesamen 728 pont, yder pont met het maecken $21 / 2$ stuyver, wel verstaende dat de kerck gelevert heeft 555 pont out loot voor I stuyver elk pont. Dies heeft hy ontfangen $63 \mathrm{gl}$ ende 5 stuyver".

Den 8sten Augustus 1593 wordt vermeld: „Aen Coen GerbrandTs voor het scheepken dat in de kerck hanght hermaeckt ro gl". Er was dus toen slechts een scheepje aanwezig. Dit blijkt ook uit de aanteekening van den 19 den September I 593: "Aen HUYGH CORNELISzOON van het scheepken te stofferen 30 stuyvers". Toen de Kerstkapel in 1556 voor rekening van PIETER vaN DORP vernieuwd was, had men haar van een balustrade met obelisken in Renaissancestijl voorzien. Van deze balustrade bleef nog een klein deel in een der steunbeeren van den noorderkruisbeuk bewaard. Reeds in I 593 was zij in slechten staat, want toen heeft Huygh Cornelisz „de crans op de nieuwe Capel over de St. Jansstraat gerepareert en twee mael met lootwit overgeverfd."

Deze balustrade werd onlangs weder op de kapel aangebracht.

Merkwaardig is een aanteekening van den 3 osten Juli I 595. „Aen de Coster van Sint Janskercke tot een geschenck gegeven voor de moeyten bij het maecken van den nieuwen thoren aldaer 3 guld," Immers hieruit blijkt, dat het sierlijke torentje, hetwelk uit het dak dier kerk rijst, toen gemaakt werd. 
Dat men in de zeventiende eeuw reeds gewoon was gehouwen steen te verwen, om haar tegen den invloed van het weder te beschermen, zagen wij hierboven. Toen in 1628 het portaal der Janskerk gereed was, werd aan "JACOB HUYBerTz voor verf ende loodwit aen de poort van de Janskerck" een belangrijk bedrag betaald.

Het portaal, dat zich aan de oostzijde van het koor der Sint-Bavokerk bevindt, heeft reeds tot velerlei gissing aanleiding gegeven. Immers vóór de Hervorming maakte men zulke ingangen niet, terwijl ook de Gothische vormen van dit portaal een zeer eigenaardig karakter hebben. Een aanteekening van den 2osten April I637 zegt: "Aen de weduwe van Dominicus JAnsz. voor verscheyde teyckeninghen van het oosterportael met kennisse van kerckmeesteren toen ter tyt 8 gl. 19 st." Hoe lang het geleden was, dat die teekeningen gemaakt werden, blijkt hieruit niet. Doch waarschijnlijk is het, dat het portaal van omstreeks 1600 moet dagteekenen.

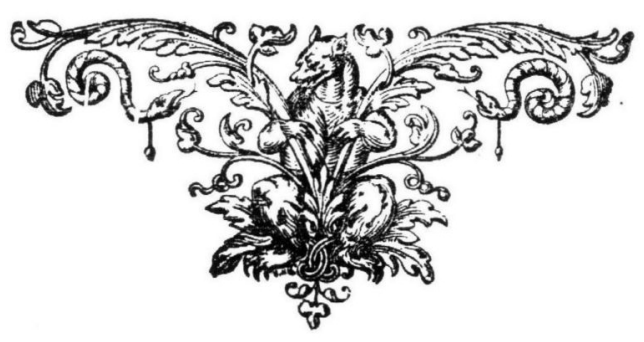

